GSA Data Repository Item \# 91-34

Title of article Age diversity of the deep crust in northern Mexico

Author(s) Roberta L. Rudnick and Kenneth L. Cameron

see Geology v. 19 , p. $1197-1200$

Contents

Table $2-2$ pg. 
Table 2. Zircon analyses. All data are ${ }^{208} \mathrm{~Pb}$ corrected unless otherwise noted.

Spot $\mathrm{U} \quad \mathrm{Th} \quad \mathrm{Th} / \mathrm{U} \quad{ }^{204} \mathrm{~Pb} \quad \mathrm{f}(\%) \quad{ }^{206} \mathrm{~Pb} / 238 \mathrm{U} \quad{ }^{207} \mathrm{~Pb} / 235 \mathrm{U} \quad{ }^{207} \mathrm{~Pb} / 206 \mathrm{~Pb}$ ppm ppm ppb

Ages (Ma)

$206 \mathrm{~Pb} / 238 \mathrm{U} 207 \mathrm{~Pb} / 206 \mathrm{~Pb}$

GNX-20 Intermediate Orthogneiss

$\begin{array}{lcccccl}1.1-\mathrm{c} & 101 & 62 & 0.612 & 5 & 7.4 & 0.1488 \pm 64 \\ 1.2-\mathrm{r}^{+} & 51 & 31 & 0.611 & 8 & 2.2 & 0.1460 \pm 64 \\ 1.3 & 101 & 63 & 0.630 & 5 & 1.0 & 0.0973 \pm 25 \\ 2.1^{+} & 105 & 91 & 0.869 & 9 & 0.8 & 0.2218 \pm 96 \\ 2.2^{+} & 130 & 123 & 0.941 & 7 & 0.5 & 0.2208 \pm 96 \\ 3.1 & 112 & 75 & 0.674 & 0 & 0 & 0.2426 \pm 105 \\ 4.1 & 119 & 107 & 0.897 & 3 & 0.3 & 0.1583 \pm 68 \\ 5.1-\mathrm{c} & 93 & 66 & 0.711 & 17 & 1.5 & 0.2536 \pm 66 \\ 5.2-\mathrm{r} & 50 & 38 & 0.765 & 26 & 26.4 & 0.0296 \pm 9 \\ 6.1+ & 112 & 108 & 0.957 & 7 & 2.4 & 0.0544 \pm 15 \\ 7.1 & 126 & 111 & 0.882 & 5 & 13.1 & 0.0053 \pm 2 \\ 8.1 & 92 & 57 & 0.617 & 4 & 0.4 & 0.2344 \pm 61 \\ 9.1 & 107 & 100 & 0.932 & 6 & 0.5 & 0.2520 \pm 65 \\ 10.1 & 115 & 101 & 0.880 & 0 & 0 & 0.1455 \pm 70 \\ 11.1 & 77 & 57 & 0.747 & 1 & 0.1 & 0.2070 \pm 101 \\ 12.1+ & 105 & 75 & 0.715 & 11 & 3.2 & 0.0632 \pm 32 \\ 13.1 & 79 & 62 & 0.789 & 0 & 0 & 0.2027 \pm 99 \\ 14.1 & 57 & 38 & 0.675 & 2 & 1.2 & 0.0467 \pm 23 \\ 15.1+ & 89 & 52 & 0.624 & 5 & 0.9 & 0.1407 \pm 70 \\ 16.1 \dagger & 90 & 77 & 0.851 & 1 & 21.0 & 0.00115 \pm 14 \\ 17.1 & 98 & 75 & 0.769 & 22 & 4.9 & 0.0894 \pm 43 \\ 18.1+ & 128 & 118 & 0.915 & 9 & 2.1 & 0.0664 \pm 32 \\ 19.1 & 148 & 148 & 1.004 & 0 & 0 & 0.1580 \pm 77 \\ 19.2+ & 119 & 105 & 0.883 & 2 & 0.5 & 0.0732 \pm 57 \\ 20.1 & 97 & 76 & 0.788 & 17 & 2.5 & 0.1388 \pm 68 \\ 20.2 & 108 & 88 & 0.814 & 0 & 0 & 0.1757 \pm 138 \\ 21.1 & 71 & 43 & 0.607 & 2 & 5.5 & 0.00995 \pm 63 \\ 21.2 & 60 & 36 & 0.597 & 1 & 1.3 & 0.0314 \pm 25 \\ 22.1+ & 102 & 76 & 0.751 & 0 & 0 & 0.1121 \pm 55 \\ 23.1 & 939 & 75 & 0.080 & 0 & 0 & 0.1711 \pm 82 \\ 23.2 & 118 & 487 & 0.242 & 8 & 0.3 & 0.1030 \pm 80 \\ 24.1 & 58 & 27 & 0.478 & 5 & 9.5 & 0.1916 \pm 70 \\ 25.1 & 77 & 56 & 0.735 & 3 & 0.6 & 0.1551 \pm 55 \\ 26.1 & 137 & 20 & 0.143 & 2 & 2.7 & 0.0104 \pm 4 \\ 27.1 & 157 & 52 & 0.333 & 2 & 0.6 & 0.0444 \pm 16 \\ 28.1 & 83 & 55 & 0.660 & 0 & 0 & 0.2044 \pm 75 \\ 29.1 & 132 & 112 & 0.848 & 0 & 0 & 0.1914 \pm 70\end{array}$

$1.558 \pm 163$

$0.997 \pm 60$

$2.642 \pm 154$

$2.558 \pm 130$

$2.995 \pm 154$

$1.778 \pm 99$

$2.626 \pm 112$

$0.461 \pm 26$

$0.054 \pm 15$

$2.738 \pm 104$

$2.579 \pm 113$

$1.450 \pm 103$

$2.571 \pm 169$

$0.683 \pm 115$

$2.486 \pm 166$

$0.647 \pm 62$

$1.487 \pm 195$

$0.948+90$

$0.511 \pm 86$

$1.655 \pm 118$

$0.748 \pm 91$

$1.401 \pm 113$

$2.184 \pm 192$

$0.0628 \pm 282$

$0.299 \pm 47$

$1.520 \pm 175$

$1.818 \pm 90$

$1.067 \pm 88$

$1.919 \pm 97$

$1.860 \pm 95$

$0.0826 \pm 86$

$0.5911 \pm 274$

$2.445 \pm 122$

$2.555 \pm 115$
$0.0724 \pm 24$

$0.0774 \pm 69$

$0.0743 \pm 38$

$0.0864 \pm 29$

$0.0840 \pm 18$

$0.0895 \pm 20$

$0.0815 \pm 25$

$0.0751 \pm 23$

$0.0924 \pm 98$

$0.0738 \pm 194$

$0.0847 \pm 21$

$0.0742 \pm 24$

$0.0723 \pm 34$

$0.0901 \pm 35$

$0.0734 \pm 121$

$0.0890 \pm 35$

$0.1004 \pm 76$

$0.0766 \pm 88$

$0.0769 \pm 58$

$0.0558 \pm 87$

$0.0759 \pm 36$

$0.0742 \pm 62$

$0.0732 \pm 43$

$0.0902 \pm 28$

$0.0458 \pm 207$

$0.0690 \pm 87$

$0.0984 \pm 96$

$0.0771 \pm 6$

$0.0752 \pm 13$

$0.0727 \pm 22$

$0.0893 \pm 28$

$0.0574 \pm 52$

$0.0965 \pm 24$

$0.0868 \pm 26$

$0.0968 \pm 22$
$894 \pm 36$

$879 \pm 36$

$599 \pm 15 \quad 1049 \pm 105$

$1291 \pm 51 \quad 1347 \pm 65$

$1286 \pm 50 \quad 1293 \pm 42$

$1400 \pm 54 \quad 1416 \pm 44$

$948 \pm 38 \quad 1232 \pm 60$

$1457 \pm 34 \quad 1071 \pm 62$

$188 \pm 6$

$341 \pm 9$

$34 \pm 1$

$1477 \pm 200$

$1309 \pm 48$

$1449 \pm 33 \quad 1047 \pm 65$

$876 \pm 40 \quad 994 \pm 95$

$1213 \pm 54 \quad 1427 \pm 73$

$395 \pm 19 \quad 1157 \pm 310$

$1190 \pm 53 \quad 1403 \pm 76$

$294 \pm 14 \quad 1632 \pm 144$

$849 \pm 40 \quad 1111 \pm 230$

$7 \pm 1$

$552 \pm 26 \quad 1120 \pm 150$

$414 \pm 20 \quad 446 \pm 313$

$946 \pm 43 \quad 1094 \pm 97$

$455 \pm 35 \quad 1046 \pm 179$

$838 \pm 38 \quad 1019 \pm 124$

$1043 \pm 76 \quad 1429 \pm 61$

$64 \pm 4$

$200 \pm 16 \quad 900 \pm 285$

$685 \pm 32 \quad 1593 \pm 195$

$1018 \pm 45 \quad 1123 \pm 17$

$632 \pm 47 \quad 1072 \pm 36$

$1130 \pm 38 \quad 1005 \pm 64$

$907 \pm 31 \quad 1410 \pm 62$

$67 \pm 3 \quad 505 \pm 190$

$280 \pm 10 \quad 1558 \pm 47$

$1199 \pm 40 \quad 1355 \pm 59$

$1129 \pm 38 \quad 1563 \pm 43$

\section{GNX-22 Paragneiss}

$\begin{array}{cccc}1.1 & 1367 & 7 & 0.005 \\ 2.1 & 1145 & 7 & 0.006 \\ 3.1 & 412 & 8 & 0.020 \\ 4.1 & 1555 & 19 & 0.012 \\ 5.1 & 610 & 29 & 0.047 \\ 6.1 & 658 & 32 & 0.049 \\ 7.1 & 537 & 29 & 0.054 \\ 8.1 & 439 & 31 & 0.070 \\ 9.1 & 915 & 37 & 0.040 \\ 10.1 & 473 & 89 & 0.188 \\ 11.1 & 396 & 83 & 0.209 \\ 12.1 & 532 & 278 & 0.522 \\ 13.1 & 415 & 2 & 0.004 \\ 14.1 & 633 & 5 & 0.008 \\ 15.1 & 775 & 58 & 0.074 \\ 16.1 & 767 & 29 & 0.038 \\ 17.1 & 659 & 90 & 0.136 \\ 18.1 & 236 & 20 & 0.086 \\ 19.1 & 179 & 69 & 0.384 \\ 20.1 & 511 & 4 & 0.008\end{array}$

$0.1 \quad 0.02897 \pm 81$

$0.2 \quad 0.01809 \pm 51$

$0.3 \quad 0.02086 \pm 59$

$0.3 \quad 0.01011 \pm 29$

$0.9 \quad 0.00652 \pm 19$

$\begin{array}{ll}1.4 & 0.00359 \pm 11\end{array}$

$2.6 \quad 0.00284 \pm 9$

$\begin{array}{ll}3.5 & 0.00271 \pm 10\end{array}$

$2.90 .00238 \pm 8$

$2.2 \quad 0.00627 \pm 19$

$3.1 \quad 0.00461 \pm 15$

$0 \quad 0.1289 \pm 36$

$\begin{array}{ll}1.1 & 0.0586 \pm 11\end{array}$

$0.3 \quad 0.04177 \pm 76$

$1.0 \quad 0.0116 \pm 2$

$3.8 \quad 0.00491 \pm 12$

0.2

0.2

0

2.7

$0.0912 \pm 17$

$0.1989 \pm 40$

$0.00508 \pm 14$
$0.1802 \pm 58$ $0.1349 \pm 46$ $0.1586 \pm 64$ $0.0670 \pm 26$ $0.0398 \pm 24$

$0.0235 \pm 18$

$0.0145 \pm 16$

$0.0170 \pm 23$

$0.0119 \pm 13$

$0.0413 \pm 33$

$0.0331 \pm 35$

$1.210 \pm 41$

$0.444 \pm 17$

$0.2921 \pm 100$

$0.1069 \pm 56$

$0.0407 \pm 42$

$0.1078 \pm 64$

$0.6101 \pm 247$

$1.914 \pm 72$

$0.0288 \pm 41$
$0.0507 \pm 14$
$0.0451 \pm 6$

$0.0541 \pm 9$

$0.0552 \pm 14$

$0.0480 \pm 12$

$0.0443 \pm 22$

$0.0474 \pm 31$

$0.0369 \pm 39$

$0.0455 \pm 58$

$0.0363 \pm 38$

$0.0478 \pm 34$

$0.0520 \pm 51$

$0.0681 \pm 11$

$0.0549 \pm 17$

$0.0667 \pm 31$

$0.061 \pm 59$

$0.0447 \pm 24$

$0.0485 \pm 16$

$184 \pm 5$

$116 \pm 3$

$133 \pm 4$

$65 \pm 2$

$42 \pm 1$

$23 \pm 1$

$18 \pm 1$

$17 \pm 1$

$15 \pm 1$

$40 \pm 1$

$30 \pm 1$

$782 \pm 21$

$367 \pm 7$

$264 \pm 5$

$75 \pm 1$

$32 \pm 1$

$112 \pm 2$

$563 \pm 10 \quad 125 \pm 76$

$0.0698 \pm 21$

$0.0411 \pm 56$
$1169+22$

$33 \pm 1$
$375 \pm 36$

$418 \pm 58$

$104 \pm 54$

$75 \pm 142$

0

$92 \pm 156$

$284 \pm 231$

$872 \pm 33$

$409 \pm 72$

$228 \pm 63$

$828 \pm 99$

$608 \pm 228$

0
\pm 76

$923 \pm 62$ 
Table 2 (continued)

Spot U Th Th/U ${ }^{204} \mathrm{~Pb} \quad \mathrm{f}(\%)$ ppm ppm ppb

${ }^{206} \mathrm{~Pb} / 238 \mathrm{U} \quad{ }^{207} \mathrm{~Pb} / 235 \mathrm{U} \quad 207 \mathrm{~Pb} / 206 \mathrm{~Pb}$

Ages (Ma)

${ }^{206} \mathrm{~Pb} / 238 \mathrm{U}{ }^{207} \mathrm{~Pb} / 206 \mathrm{~Pb}$

MN-19 Intermediate Orthogneiss

$\begin{array}{lcccccl}1.1 & 410 & 2 & 0.005 & 2 & 3.4 & 0.00262 \pm 9 \\ 2.1 \dagger & 57 & 42 & 0.724 & 7 & 30.4 & 0.00642 \pm 30 \\ 3.1 & 104 & 55 & 0.524 & 0 & 0 & 0.04216 \pm 99 \\ 4.1 & 282 & 221 & 0.783 & 0 & 0 & 0.02750 \pm 65 \\ 5.1 & 203 & 169 & 0.834 & 0 & 0 & 0.05217 \pm 122 \\ 6.1 & 135 & 55 & 0.411 & 0 & 0 & 0.02806 \pm 67 \\ 7.1 & 316 & 326 & 1.033 & 0 & 0 & 0.04969 \pm 116 \\ 8.1 & 443 & 86 & 0.194 & 1 & 0.2 & 0.02861 \pm 67 \\ 9.1 & 364 & 297 & 0.817 & 0 & 0 & 0.05628 \pm 131 \\ 10.1 \dagger & 349 & 109 & 0.313 & 0 & 0 & 0.00155 \pm 8 \\ 11.1+59 & 37 & 0.621 & 0 & 0 & 0.00583 \pm 28 \\ 12.1 & 554 & 647 & 1.167 & 0 & 0 & 0.04523 \pm 105 \\ 13.1 & 523 & 184 & 0.351 & 1 & 0.1 & 0.02558 \pm 60 \\ 14.1 \dagger 1691 & 899 & 0.532 & 1 & 4.0 & 0.00032 \pm 2 \\ 15.1 & 141 & 78 & 0.556 & 0 & 0 & 0.03972 \pm 94 \\ 16.1 & 630 & 738 & 1.171 & 0 & 0 & 0.04789 \pm 112 \\ 17.1 & 421 & 443 & 1.052 & 0 & 0 & 0.03280 \pm 78 \\ 18.1+ & 160 & 50 & 0.314 & 1 & 0.6 & 0.03122 \pm 75\end{array}$

$0.0172 \pm 27 \quad 0.0476 \pm 70$

$17 \pm 1$

$85 \pm_{85}^{312}$

$0.3414 \pm 248 \quad 0.0587 \pm 39$

$0.1916 \pm 146 \quad 0.0505 \pm 35$

$0.3846 \pm 266 \quad 0.0535 \pm 33$

$0.2108 \pm 162 \quad 0.0545 \pm 38$

$0.3609 \pm 260 \quad 0.0527 \pm 34$

$0.2168 \pm 96 \quad 0.0549 \pm 19$

$0.4271 \pm 237 \quad 0.0550 \pm 26$

$41 \pm 2$

$266 \pm 6$

$175 \pm 4$

$328 \pm 7$

$178 \pm 4$

$313 \pm 7$

$182 \pm 4$

$353 \pm 8$

$10 \pm 0$

$0.0765 \pm 103 \quad 0.0951 \pm 114$

$37 \pm 2$

$0.3756 \pm 225 \quad 0.0602 \pm 32$

$285 \pm 7$

$0.1609 \pm 82 \quad 0.0456 \pm 19$

$163 \pm 4$

$2 \pm 0$

$0.2874 \pm 231 \quad 0.0525 \pm 39$

$251 \pm 6$

$302 \pm 7$

$0.4124 \pm 258 \quad 0.0624 \pm 34$

$0.2584 \pm 207 \quad 0.0571 \pm 42$

$208 \pm 5$

$0.2599 \pm 230 \quad 0.0604 \pm 50$

$198 \pm 5$

$557 \pm 151$

$220 \pm 162$

$348 \pm 147$

$391 \pm 165$

$315 \pm 155$

$410 \pm 80$

$414 \pm 110$

$1531 \pm 245$

$612 \pm 118$

0

$306 \pm 176$

$689 \pm 1$

$497 \pm 171$

MN-40 Intermediate Orthogneiss*

$\begin{array}{lccc}1.1 & 563 & 74 & 0.130 \\ 2.1 & 63 & 62 & 0.980 \\ 3.1 & 60 & 40 & 0.666 \\ 4.1 & 563 & 567 & 1.007 \\ 5.1 & 156 & 77 & 0.494 \\ 6.1 & 195 & 116 & 0.592 \\ 7.1 & 379 & 234 & 0.617 \\ 8.1-c & 147 & 68 & 0.466 \\ 8.2-\mathrm{r} & 199 & 123 & 0.617 \\ 9.1 & 182 & 122 & 0.671 \\ 10.1 & 127 & 117 & 0.918 \\ 11.1 & 73 & 45 & 0.620 \\ 12.1 & 205 & 105 & 0.514 \\ 13.1 & 137 & 89 & 0.649 \\ 14.1 & 142 & 85 & 0.600 \\ 15.1 & 152 & 122 & 0.799 \\ 16.1 & 360 & 271 & 0.755 \\ 17.1 & 647 & 171 & 0.264\end{array}$

$0.00596 \pm 18$

$0.00511 \pm 25$

$0.00695 \pm 29$

$0.00586 \pm 18$

$0.00566 \pm 21$

$0.00557 \pm 21$

$0.00444 \pm 15$

$0.03057 \pm 87$

$0.00592 \pm 20$

$0.00627 \pm 22$

$0.00428 \pm 19$

$0.00561 \pm 40$

$0.00622 \pm 35$

$0.00552 \pm 34$

$0.00610 \pm 36$

$0.00558 \pm 33$

$0.00524 \pm 28$

$0.00395 \pm 21$
$0.5451 \pm 274 \quad 0.0663 \pm 24$

$0.1559 \pm 160 \quad 0.2211 \pm 187$

$0.1804 \pm 167 \quad 0.1884 \pm 146$

$0.0559 \pm 28 \quad 0.0692 \pm 25$

$0.1158 \pm 85 \quad 0.1484 \pm 88$

$0.0870 \pm 69 \quad 0.1133 \pm 74$

$0.0504 \pm 32 \quad 0.0824 \pm 42$

$0.2603 \pm 124 \quad 0.0618 \pm 22$

$0.0737 \pm 53 \quad 0.0903 \pm 53$

$0.0858 \pm 64 \quad 0.0992 \pm 61$

$0.0746 \pm 73 \quad 0.1264 \pm 104$

$0.2180 \pm 321 \quad 0.2818 \pm 339$

$0.1074 \pm 122 \quad 0.1252 \pm 116$

$0.1697 \pm 206 \quad 0.2232 \pm 217$

$0.1551 \pm 185 \quad 0.1843 \pm 177$

$0.1254 \pm 145 \quad 0.1631 \pm 152$

$0.0805 \pm 82 \quad 0.1114 \pm 89$

$0.0478 \pm 45 \quad 0.0878 \pm 64$

\section{MN-20 Mafic Granulite ${ }^{\dagger}$}

$\begin{array}{lcccccl}1.1 & 78 & 42 & 0.534 & 8 & 90.9 & 0.00020 \pm 13 \\ 1.2 & 84 & 63 & 0.746 & 4 & 67.9 & 0.00038 \pm 8 \\ 2.1 & 133 & 76 & 0.574 & 42 & 93.0 & 0.00044 \pm 19 \\ 2.2 & 53 & 40 & 0.752 & 2 & 93.7 & 0.00004 \pm 8 \\ 4.1 & 63 & 37 & 0.585 & 5 & 95.6 & 0.00006 \pm 13 \\ 5.1 & 60 & 60 & 1.004 & 4 & 94.0 & 0.00008 \pm 13 \\ 3.1 & 73 & 47 & 0.647 & 4 & 73.8 & 0.00036 \pm 11 \\ 6.1 & 111 & 80 & 0.718 & 5 & 89.6 & 0.00010 \pm 10 \\ 7.1 & 46 & 32 & 0.697 & 5 & 98.7 & 0.00003 \pm 23 \\ 8.1 & 53 & 27 & 0.519 & 10 & 84.4 & 0.00063 \pm 32 \\ 8.2 & 41 & 24 & 0.584 & 22 & 84.5 & 0.00183 \pm 45\end{array}$

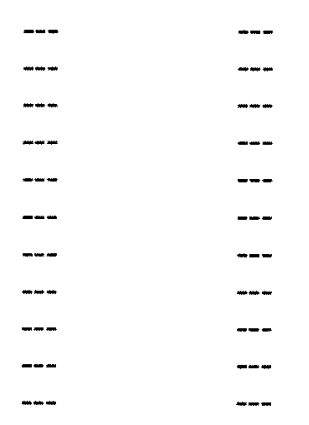

$\begin{array}{cc}1 \pm 1 & -- \\ 2 \pm 1 & -- \\ 3 \pm 1 & -- \\ 0 & -- \\ 0 & -- \\ 0 & -- \\ 2 \pm 1 & -- \\ 1 \pm 1 & -- \\ 0 & - \\ 4 \pm 2 & - \\ 12 \pm 3 & --\end{array}$

All errors are 1 sigma. $\mathrm{c}=$ core, $\mathrm{r}=\mathrm{rim}$.

tData corrected using $207 \mathrm{~Pb}$.

+Data corrected using ${ }^{204} \mathrm{~Pb}$.

*Data uncorrected for common $\mathrm{Pb}$. 